\title{
Article \\ 2D Omni-Directional Wireless Power Transfer Modeling for Unmanned Aerial Vehicles with Noncollaborative Charging System Control
}

\author{
Oussama Allama ${ }^{1}$, Mohamed Hadi Habaebi ${ }^{1, * \mathbb{D}}$, Sheroz Khan ${ }^{2} \mathbb{D}$, Elfatih A. A. Elsheikh ${ }^{3}$ and \\ Fakher Eldin M. Suliman ${ }^{3}$
}

check for

updates

Citation: Allama, O.; Habaebi, M.H.; Khan, S.; Elsheikh, E.A.A.; Suliman, F.E.M. 2D Omni-Directional Wireless Power Transfer Modeling for Unmanned Aerial Vehicles with Noncollaborative Charging System Control. Electronics 2021, 10, 2858. https://doi.org/10.3390/

electronics10222858

Academic Editor: Ahmed Abu-Siada

Received: 10 October 2021

Accepted: 17 November 2021

Published: 19 November 2021

Publisher's Note: MDPI stays neutra with regard to jurisdictional claims in published maps and institutional affiliations.

Copyright: (C) 2021 by the authors Licensee MDPI, Basel, Switzerland. This article is an open access article distributed under the terms and conditions of the Creative Commons Attribution (CC BY) license (https:// creativecommons.org/licenses/by/ $4.0 /)$.
1 IoT and Wireless Communication Protocols Laboratory, Department of Electrical and Computer Engineering, International Islamic University Malaysia (IIUM), Kuala Lumpur 53100, Malaysia; allama.oussama@live.iium.edu.my

2 Department of Electrical Engineering, College of Engineering and Information Technology, Unaizah Colleges, Unaizah 2053, Saudi Arabia; sheroz@oc.edu.sa

3 Department of Electrical Engineering, College of Engineering, King Khalid University, Abha 61421, Saudi Arabia; eelsheikh@kku.edu.sa (E.A.A.E.); fmsuliman@kku.edu.sa (F.E.M.S.)

* Correspondence: habaebi@iium.edu.my

Abstract: Wireless power transfer (WPT) has been extensively studied from various aspects such as far field and near field, operating frequency, coil design, matched capacitance values, misaligned locations of transmitting and receiving coils, distance variance between them, target loads in the specific locations, environment, and operating conditions. This is due to the usefulness of WPT technology in many applications, including the revolutionary method of auto-recharging of unmanned aerial vehicles (UAVs). This paper presents analytical modeling of a WPT-link with two orthogonal transmitting coils arranged to produce an omnidirectional magnetic field suitable for charging a moving rotating load, maximizing energy transfer without any feedback from the receiving end. To achieve a suitable 2D WPT simulation system, as well as an accurate control design, the mutual coupling values in terms of receiver angular rotation are simulated using Ansys software. Power transfer is maximized by using extremum seeking control (ESC), making use of the input power as an objective function with specific parameter values that represent the WPT model to obtain the results. The results shown are those of the input power transmitted by the transmitting-end coils to a load of an orbiting mobile UAV. Based on the simulation results, the controller can achieve maximum power transfer in $100 \mu \mathrm{s}$ of duration when the speed of the UAV is close to $314 \mathrm{rad} / \mathrm{s}$.

Keywords: 2D omnidirectional; WPT maximization; extremum seeking control; tracking

\section{Introduction}

Unmanned aerial vehicles (UAVs) are commonly used for real-time crowd monitoring, extreme sports, hazardous situations rescues, delivery services, and agriculture technology applications. Maximum take-off weight (MTOW), flight time, and speed depend on battery power, which makes it one of the major limitations of UAVs. Wireless power transfer has been an interesting technology in providing the convenience of charging a large variety of devices in both industrial and the quotidian domains due to the simplicity of design by eliminating the need of using cables and batteries. WPT is an attractive battery-less solution for the Internet of Things (IoT), where a network of devices share data wirelessly. This is achieved by converting electrical energy into other relay forms of energy such as electromagnetic field energy, lasers, microwaves, and vibrations through the use of transmitters. In the inductive coupling approach, for example, electrical energy on the transmitting end is converted into magnetic relay energy that in turn is changed into electrical energy on the receiving end. After an appropriate transmission distance, the relay forms of energy are retrieved by the receiver using so-called inductive coupling. 
WPT through inductive coupling reduces the size of sensors and electronic devices (by eliminating the need for a power supply circuit or batteries), giving more flexibility in terms of sensor design and scalability, especially in smart industrial facilities and IoTintegrated smart city infrastructures [1]. It is a revolutionary method for automating the recharging process for unmanned aerial vehicles (UAVs) or drones, especially drones used in agriculture [2], crowd management [3], monitoring of traffic in major cities [4], and disaster management services [5].

Despite being light vehicles, drones experience problems with battery and flight time. However, the main drawback of WPT technology via inductive coupling is maintaining a high rate of wireless power transmission efficiency, especially in the case of moving loads. A lot of research work has been conducted to address these issues, and several coil-based topologies have been studied, trying shapes and designs for transmitting-end and the receiving-end coils. In robot applications, a three orthogonal coil structure was presented to pick up wireless power, in order to ensure a maximum coupling coefficient when the robot arm rotates, the primary and secondary coils are designed to fit inside the robot elbow [6]. Similarly, a WPT energy harvester with a multicoil structure was designed to solve the misalignment issue for mobile devices [7]. Additionally, in [8], a cubic magnetic dipole coil structure was proposed to transmit power omnidirectionally, and for multiloads, another research presented a 3D wireless charging cylinder based on two orthogonal coils. The system is able to generate a rotating magnetic field for charging multiple loads [9]. The work in [10] presents bowl-shaped multiple transmitting coils. The system can provide a uniform magnetic field inside the charging area to overcome the misalignment issue. In order to enhance the efficiency of an omnidirectional WPT system using a three orthogonal coil structure, the authors in [11] presented a modified design of the terminal receiver. According to the experimental results, the load power could be improved up to $240 \%$ due to the quadrature-shaped pickup receiving-coil structure. In [12], three orthogonal coil topologies are used to produce a 3D magnetic field via an inductive technique to link the source coil to multiple receivers deployed arbitrarily in the magnetic field range of the transmitter. An iterative algorithm was developed to modulate the resulting magnetic field of the three transmission coils to associate them with a specific target point with large magnetic coupling. However, the downside caused by scaling the charge to multiple loads in the rotational WPT is that the efficiency of the WPT approach will be reduced due to magnetic field leakage issues $[8,12]$. To overcome the above obstacles and contribute to the development of WPT, technology researchers decided to put forward the possibility of beaming the magnetic field to the target site. This new concept allows directional control of the resulting magnetic field by adopting a certain topology at the transmitting end, thus reducing the magnetic flux leakage problem and increasing the efficiency when compared to the rotational WPT [13]. In [14], the target load is magnetically tracked by an energy beaming approach using a crossed antenna structure at the transmitting end using a resonant coupling mechanism. The authors introduced the possibility of directing the omnidirectional magnetic field towards a specific location by controlling the current strength in the design of crossed antenna structure at the transmitting end. Several models have been proposed to maximize power delivery in the omnidirectional power transfer model. In [15], the generation of the rotating magnetic field is controlled by varying the current amplitude characteristics of the three orthogonal transmission coils. Since the current amplitude of each coil is different from the other, and the resultant currents are constant, the output vector of the rotating magnetic field can be generated at any specific point of the spherical region. To maximize the efficiency of power transmission, the paper proposed two approaches, the mathematical method and the two-dimensional plane method. However, both methods ignore the receiver navigation and are free from using a closed-loop time response. In [16], the same theory is coupled with the control technique of [15]. Each vector fired to a certain point in space is recorded, and the values of the three amplitude currents of each coil are determined, as well as the magnetic resultant angles. Then, the power vector with respect to the point is determined 
using a weighted time-sharing technique. Thus, the magnetic field focuses on the loaded area. However, this study also only focused on static loads; thus, it requires large input data to scan, analyze, and compare the parameters of each point surrounding the sphere, which requires a lot of resources and makes it unsuitable for applications in smart systems. In [17], other control methods are used to achieve higher efficiency, but the research does not take into account the load mobility, and calibration in three different modes of the receiver is needed before starting the power control process. Omnidirectional WPT of a moving load target is reported in [18], using the gradient descent technique. However, due to the continuous improvement of this technology, a large number of calculations that require a powerful and expensive processor are applied. Furthermore, the speed and trajectory of movement are not discussed in the paper.

The contribution of this paper lies in the development of the detailed analytical model and control design for a 2D WPT system aimed at powering a moving receiving load rotating on an orbital path. The rotational mutual coupling for the $2 \mathrm{D}$ system is generated based on the Ansys software, which is used in the power simulation, and the results match with those in the benchmark paper [17]. By deriving the expression for mutual coupling in terms of the receiving angle, the mobile load movement can be simulated by including its velocity, thus observing the effect of the receiver movement on the input power. Moreover, the derived expression allows for fine tuning of the controller and closed-loop response analysis. We developed an accurate 2D WPT model system that can accurately simulate the receiver navigation (trajectory and velocity), considering the distance between the transmitting-end and the receiving-end coils. Ultimately, the designed controller can maximize the power transfer regardless of the variations in position and velocity, as well as the distance to the load. The novelty of this work lies in deriving the expression of the mutual coupling in terms of the receiver trajectory, where the motion is rotational and continuous, and the input power of the 2D system in terms of the receiving angle. Furthermore, the extremum seeking control technique was used to maximize power transmission when the receiver rotates at high speed. The simulation results for this work are validated with the experimental results in [17]. The proposed model can simulate an average angular velocity of $314 \mathrm{rad} / \mathrm{s}$, and the designed controller is able to provide maximum power delivery with a response time of less than $100 \mu \mathrm{s}$. In order to test the effectiveness of the controller, several variable-speed receiving-load paths were simulated using MATLAB, yielding results consistent with the theoretical interpretation.

The rest of the paper is organized as follows: Section 2 contains an analysis of the parameters of the two-dimensional WPT system, including a derivation of the relationship between the input power and the location of the mobile receiver. Section 3 explains extremum seeking control (ES) algorithms, as well as the mobile receiver tracking process as a result of Section 2. In Section 4, the effect of the change in distance $d$ between the receiver and the transmitter on the two-dimensional WPT system is studied. Section 5 concludes the paper as a quantitative conclusion of this research.

\section{Mathematical Analysis of 2D WPT System}

\subsection{System Modeling}

The system consists of two larger-sized orthogonally arranged transmitting-end coils $\left(T_{x}, T_{y}\right)$ and one smaller-sized centrally aligned receiving-end coil, as shown in Figure 1. The distance between the centers is assumed to be constant, but the receiving end has rotational motion moving at variable speed in the $(x, y)$ plane. Each coil is connected to a serial capacitor and resistor to make a resonant circuit, which increases the efficiency of the WPT. The transmission side is provided by two separate alternating-current (AC) sources to drive the power transmission, while the receiving coil is connected to a resistive load. 


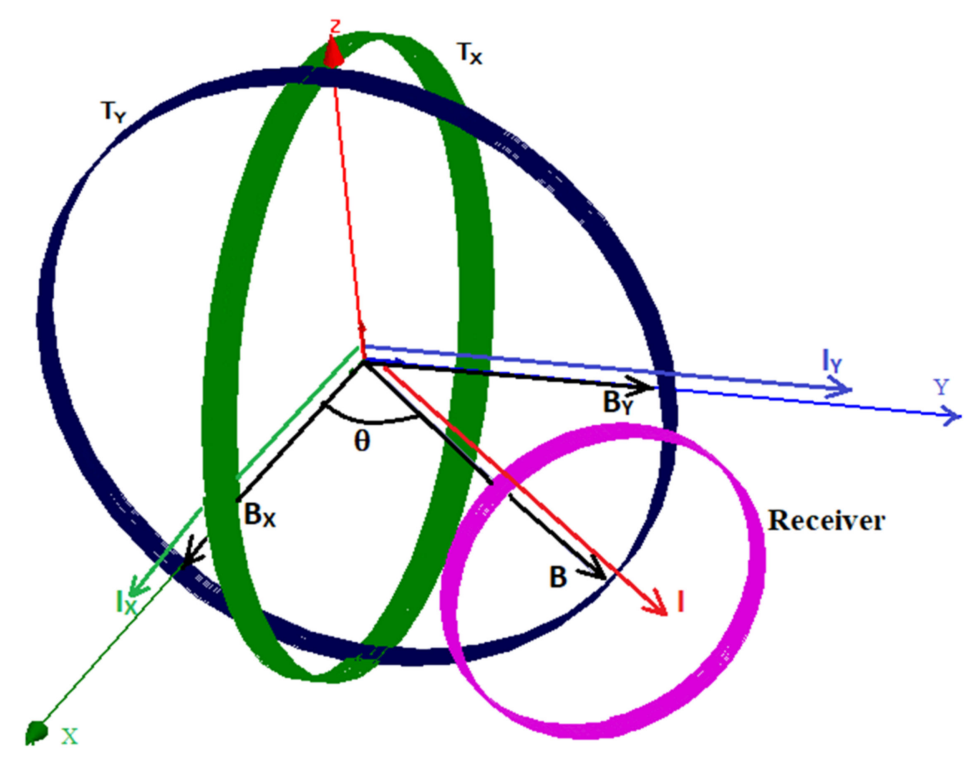

Figure 1. Two-dimensional (2D) WPT system magnetic and electric resultant vector.

We define the currents flowing in each transmitting coil as $i_{x} i_{y}$, producing a magnetic field $B_{x} B_{y}$, respectively. The resultant current vector $I$, and the resultant magnetic field vector $B$ of the two transmitting-end coils are related as derived in [19].

$$
B=\frac{\mu_{0} I}{4 \pi r^{2}} \oint d l \cdot(\hat{i} \times \hat{r})=\frac{\mu_{0} I}{2 r} \cdot(\hat{i} \times \hat{r})
$$

According to the Biot-Savart law [16], the vector magnetic field produced by a current flowing in a circular coil is always perpendicular to the plane of the coil and proportional to the current that creates it. Hence, by monitoring the current, we can control the magnetic field. Since the two coils are orthogonal, the resultant magnetic field is given as:

$$
B=B_{x}+B_{y}=\frac{\mu_{0}}{2 r} \cdot\left(i_{x} \hat{i}+i_{y} \hat{j}\right)
$$

From Equation (2), we can understand that the components of the magnetic field resultant vector $B$, as well as the current resultant vectors $I$, have the same direction as the unity vectors of the Cartesian plane. Therefore, it is possible to direct the magnetic field without moving the transmitter. This feature allows for the formation of the directive magnetic field by adjusting the current amplitude towards any intended point in the Cartesian plane. We define $\theta$ as the angular direction of both the magnetic and the resultant electric vector produced by the transmitting-end coils of the WPT system. Equation (3) describes the relationship between the resultants and the currents $i_{x} i_{y}$ flowing through the two sending coils $\mathrm{T}_{\mathrm{x}}$, and $\mathrm{T}_{\mathrm{y}_{\underline{L}}}$, respectively.

$$
\left\{\begin{array}{l}
i_{x}=I \times \cos \theta \\
i_{y}=I \times \sin \theta
\end{array}\right.
$$

Figure 1 shows the resulting magnetic and electric field vectors and their results in the $(x, y)$ plane. Here, we notice that by changing $\theta$, one can point the resulting magnetic field vector toward any point in the $(x, y)$ plane, which means targeting any load in the plane. For further understanding and analysis of the 2D WPT phenomenon, we present the equivalent circuit modeling of the system, as illustrated in Figure 2. 


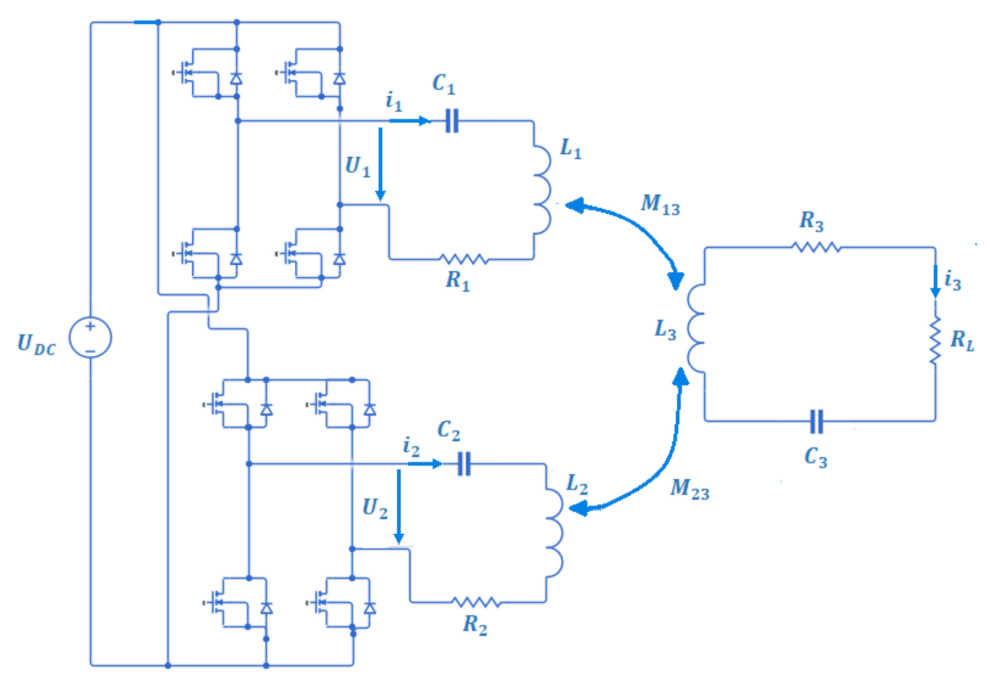

Figure 2. Equivalent circuit modeling of the 2D WPT system.

Throughout the rest of the paper, the electric circuit currents flowing in the transmitting coils $\left(\mathrm{T}_{\mathrm{x}}\right.$ and $\left.\mathrm{T}_{\mathrm{y}}\right)$ are replaced by $i_{1}$ and $i_{2}$, respectively. The electric parameters that describe the system are related mathematically by Equation (4)

$$
\left\{\begin{array}{c}
i_{1}\left(R_{1}+j w L_{1}+\frac{1}{j w C_{1}}\right)-j w M_{13} i_{3}=U_{1} \\
i_{2}\left(R_{2}+j w L_{2}+\frac{1}{j w C_{2}}\right)-j w M_{23} i_{3}=U_{2} \\
i_{3}\left(R_{3}+R_{L}+j w L_{3}+\frac{1}{j w C_{3}}\right)-j w M_{13} i_{1}-j w M_{23} i_{2}=0
\end{array}\right.
$$

$U_{1}$ and $U_{2}$ are the alternating voltage produced by the inverter blocks of Figure 2 and therefore cause the currents $i_{1}$ and $i_{2}$ to flow in the transmitting coils $\left(T_{\mathrm{x}}\right.$ and $\left.\mathrm{T}_{\mathrm{y}}\right)$, respectively, while $i_{3}$ is the current induced in the receiving load. The mutual coupling between the transmitting end and the receiving end is quantified by two values $M_{13}$ and $M_{23}$ for coils $\mathrm{T}_{\mathrm{x}}$ and $\mathrm{T}_{\mathrm{y}}$ with respect to the receiver. The sending coils are orthogonal so that their mutual coupling is cancelled. For simplicity, the self-inductances $\left(L_{1}, L_{2}\right)$, the resistors $\left(R_{1}, R_{2}\right)$, and the capacitances $\left(C_{1}, C_{2}\right)$ of the two sending loops are chosen to be equal. In order to control the magnetic field resultant vector, we must introduce the angular direction parameter $\theta$, as well as the resultant electrical vector $I$ by substituting (3) into (4) to become (5):

$$
X_{3}=\left(w L_{3}-\frac{1}{j w C_{3}}\right)\left\{\begin{array}{c}
I \cos \theta\left(R_{1}+j w L_{1}+\frac{1}{j w C_{1}}\right)-j w M_{13} i_{3}=U_{1} \\
I \sin \theta\left(R_{2}+j w L_{2}+\frac{1}{j w C_{2}}\right)-j w M_{23} i_{3}=U_{2} \\
i_{3}\left(R_{3}+R_{L}+j w L_{3}+\frac{1}{j w C_{3}}\right)-j w M_{13} I \cos \theta-j w M_{23} I \sin \theta=0
\end{array}\right.
$$

Equation (5) represents important information on the load current and power in terms of the angular direction $\theta$; hence, we can study the effect of this parameter on maximizing the power transfer efficiency through current $i_{3}$ given in (6).

$$
i_{3}=\frac{j w M_{13} I \cos \theta+j w M_{23} I \sin \theta}{\sqrt{\left(R_{3}+R_{L}\right)^{2}+X_{3}^{2}}}=\frac{j w I\left(M_{13} \cos \theta+M_{23} \sin \theta\right)}{\sqrt{\left(R_{3}+R_{L}\right)^{2}+X_{3}^{2}}}
$$

where the load current $i_{3}$ when the magnetic field rotates around the origin and the receiver placed at $135^{\circ}$ is given as shown in Figure 3. 


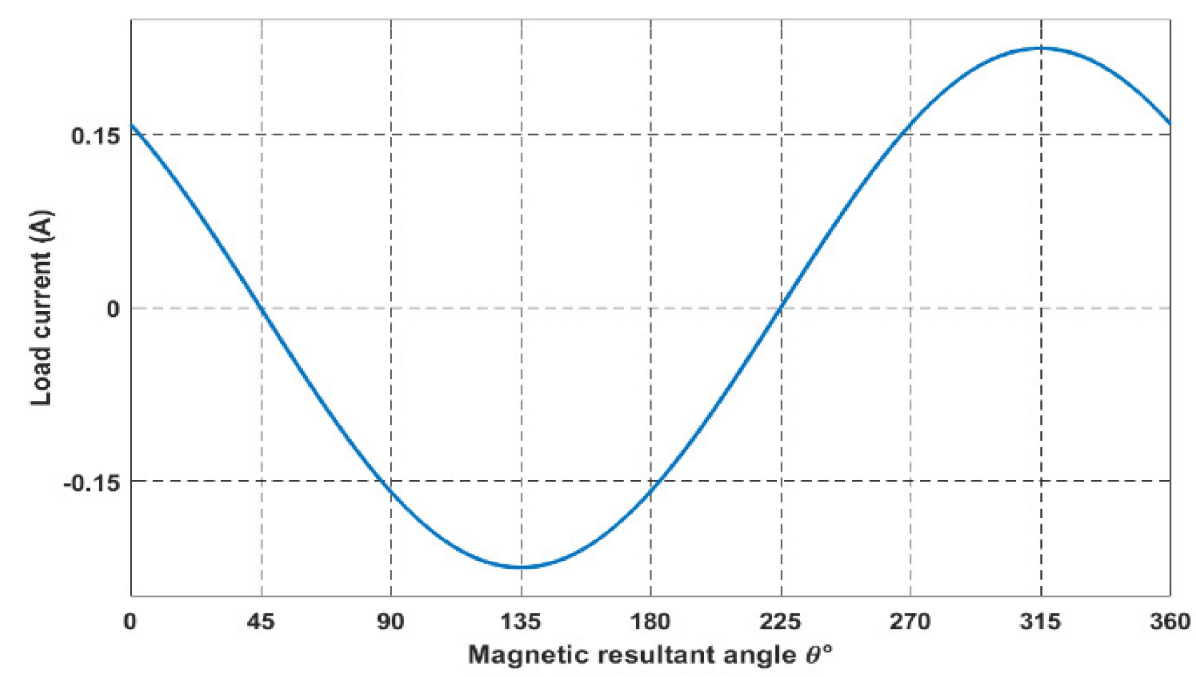

Figure 3. Load current when the magnetic field rotates around the origin for receiver placed at $\theta=135^{\circ}$.

\subsection{Deriving the Mutual Coupling Functions $M_{13}(\alpha)$ and $M_{23}(\alpha)$ in Terms of the Rotational Angle $\alpha$ of the Receiver}

In order to simulate the load current and the output power versus the angular load rotation $\alpha^{\circ}$, the values of the mutual coupling $M_{13}$ and $M_{23}$ are calculated via Ansys software simulation. Each angular position of the pink receiver generates two mutual coupling values $M_{13}$ and $M_{23}$; hence, in total, we have 360 values for each by choosing a $1^{\circ}$ rotational step change. The rotational movement of the receiver is shown in Figure 4.

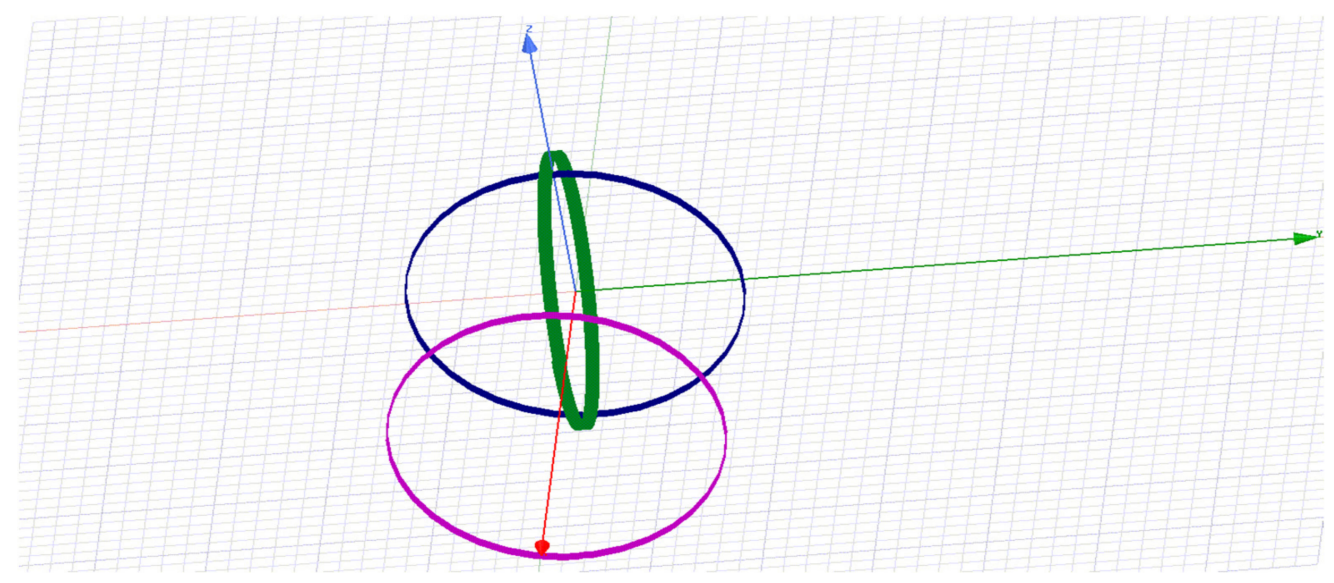

Figure 4. Two-dimensional (2D) WPT Ansys software simulation when the receiver rotates in the range of $0^{\circ}$ to $360^{\circ}$.

In order to accomplish an accurate comparison with the benchmarking papers, the parameters of the system used in the Ansys software simulation are the ones used in [17]. The two transmitting coils diameters and the receiver coil diameter are $d 1=d 2=d 3=0.3 \mathrm{~m}$, the number of turns in the transmitter coils are $T_{x}=11$ and $T_{y}=11$, the self-inductance values of the two transmitting coils are $\mathrm{L}_{1}=82.03 \mu \mathrm{H}$, and $\mathrm{L}_{2}=82.03 \mu \mathrm{H}$, the number of turns of the receiver coil is $\mathrm{T}_{3}=11$, the self-inductance of the receiver coil is $\mathrm{L} 3=82.03 \mu \mathrm{H}$, $C_{1}=C_{2}=C_{3}=1 \eta F$, the transmitter coil circuit resistances are $R_{1}=R_{2}=0.9 \Omega$, the receiver coil circuit resistance is $R_{3}=0.9 \Omega$, the load resistance is $R_{L}=10 \Omega$, the distance between the transmitters' center and the receiver's center is $d=0.3 \mathrm{~m}$, the frequency $\mathrm{f}=535 \mathrm{kHz}$, and the transmitter coil current magnitude is $0.15 \mathrm{~A}$.

Figure 5 shows the mutual coupling in terms of the receiving rotation angle $\alpha$. 


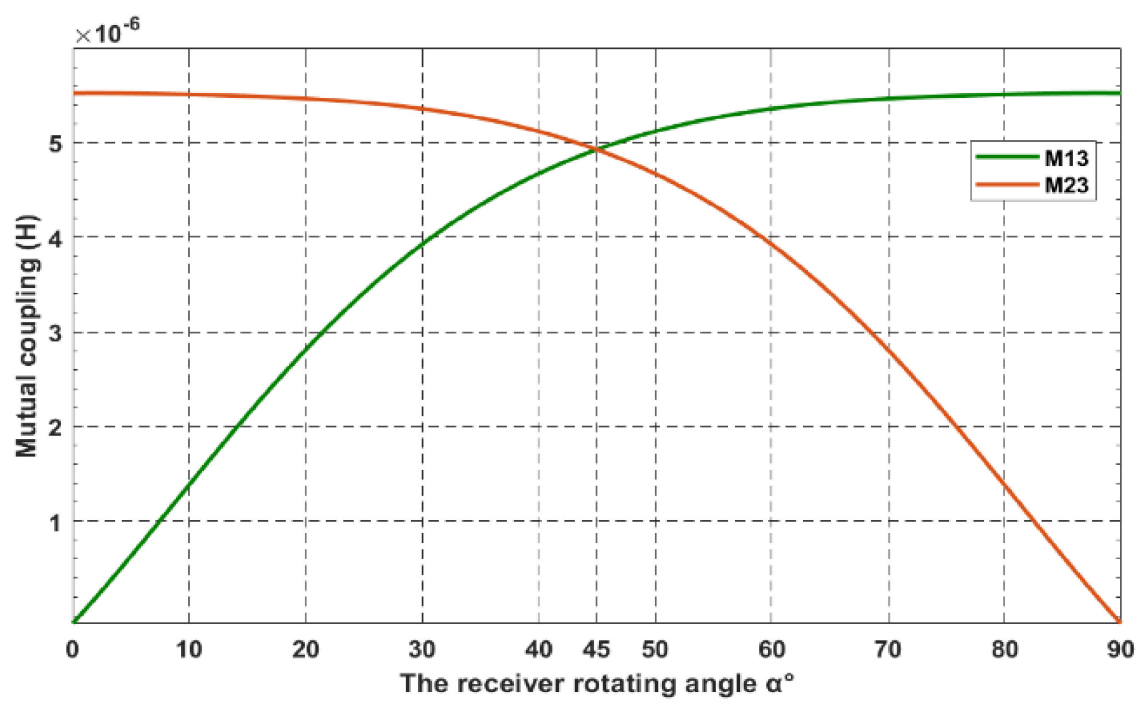

Figure 5. Mutual coupling when the receiver coil distance is fixed at $d=0.3 \mathrm{~m}$ and the angle $\alpha$ rotates from $0^{\circ}$ to $360^{\circ}$ through $1^{\circ}$ step change.

Using the MATLAB fitting tool, Figure 5 can be approximated using Fourier transform, as shown in Equations (7) and (8).

Let

$$
\begin{gathered}
M_{13}(\alpha)=a_{01}+a_{11} \cos \alpha \omega+b_{11} \sin \alpha \omega+a_{21} \cos 2 \alpha \omega \\
+b_{21} \sin 2 \alpha \omega+a_{31} \cos 3 \alpha \omega+b_{31} \sin 3 \alpha \omega \\
M_{23}(\alpha)=a_{02}+a_{12} \cos \alpha \omega+b_{12} \sin \alpha \omega+a_{22} \cos 2 \alpha \omega \\
+b_{22} \sin 2 \alpha \omega+a_{32} \cos 3 \alpha \omega+b_{32} \sin 3 \alpha \omega
\end{gathered}
$$

where $a_{01}, a_{11}, b_{11}, a_{21}, b_{21}, a_{31}, b_{31}, a_{02}, a_{12}, b_{12}, a_{22}, b_{22}, a_{32}, b_{32}, \omega$ are the Fourier constants.

By substituting (7) and (8) in the electric Equation (6) of the load current of the $2 \mathrm{D}$ system, we can derive expressions that describe the input power, the load current, and the power load in terms of the magnetic resultant angle and the angular position of the receiver $(\alpha, \theta)$; hence, the complexity imposed by the mutual coupling terms can be solved by replacing $M_{13} M_{23}$ with their Fourier expressions (7) and (8), $M_{13}(\alpha)$ and $M_{23}(\alpha)$, respectively, thus enabling an accurate control design and simulation for both the $2 \mathrm{D}$ and 3D orthogonal WPT systems.

\subsection{The 2D Angular Input Power Calculation}

By substituting (7) and (8) in (6), the load current expression becomes:

$$
i_{3}=\frac{j w I\left(M_{13}(\alpha) \cos \theta+M_{23}(\alpha) \sin \theta\right)}{\sqrt{\left(R_{3}+R_{L}\right)^{2}+X_{3}^{2}}}
$$

The power load expression is defined as follows:

$$
P_{L}=\left|R_{L}\right| \times\left|i_{3}\right|^{2}
$$

The amplitude of $i_{3}$ is as follows:

$$
\left|i_{3}\right|=\frac{w I}{\sqrt{\left(R_{3}+R_{L}\right)^{2}+X_{3}^{2}}} \times\left(M_{13}(\alpha) \cos \theta+M_{23}(\alpha) \sin \theta\right)
$$


By substituting (11) in (10), we have the final expression of the load power:

$$
P_{L}=\frac{w^{2} I^{2}}{\left(R_{3}+R_{L}\right)^{2}+X_{3}^{2}} \times\left(M_{13}(\alpha) \cos \theta+M_{23}(\alpha) \sin \theta\right)^{2}
$$

Hence, the plot of the load power expression is shown bellow.

The load power represented in Figure 6 represents a Lemniscate of Bernoulli that has two symmetric maxima.

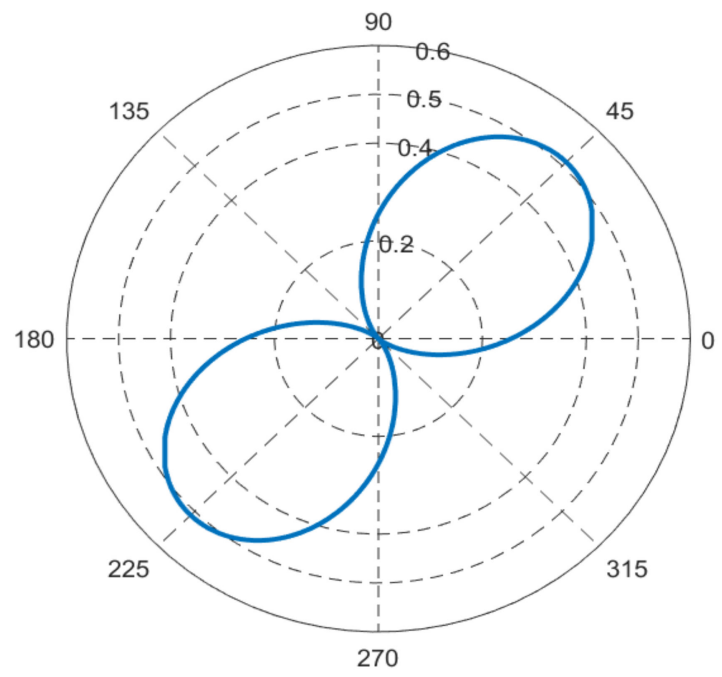

Figure 6. Polar plot of the load power when the receiver is placed at $45^{\circ}$ in the presence of a rotating magnetic field.

The input power is defined as follows:

$$
P_{\text {in }}=P_{l o s}+P_{l o s}+P_{l o s} 3+P_{L}
$$

where:

$$
\begin{gathered}
P_{\text {los } 1}=R_{1} \times i_{x}^{2}=R_{1}(I \cos \theta)^{2} \\
P_{l o s 2}=R_{2} \times i_{y}^{2}=R_{2}(I \sin \theta)^{2} \\
P_{l o s}=R_{3} \times i_{3}^{2} \\
P_{\text {in }}=R_{1}(I \cos \theta)^{2}+R_{2}(I \sin \theta)^{2}+R_{3} \times i_{3}^{2}+R_{L} \times i_{3}^{2}
\end{gathered}
$$

Let $R_{1}=R_{2}$. Substituting (9) into (15), we have:

$$
P_{\text {in }}=R I^{2}+\left(R_{3}+R_{L}\right) \frac{w^{2} I^{2}}{\left(R_{3}+R_{L}\right)^{2}+X_{3}^{2}} \times\left(M_{13}(\alpha) \cos \theta+M_{23}(\alpha) \sin \theta\right)^{2}
$$

Using (16) we can simulate the input power curve when the receiver rotates across the transmitting end, as shown in Figure 7.

The new input power expression (16) allows the control designer to simulate the continued rotational movement of the receiver by introducing the new parameter $\alpha$, in the same time, we get rid of the mutual coupling parameter, as demonstrated in this section.

Figure 8 shows the input power is a deformed Lemniscate of the Bernoulli function, and it has two maxima depending on the magnetic field resultant orientation and the position of the receiver. By varying, $\theta$ to point at the exact position of the receiver, we can maximize the WPT efficiency. However, all feedback is eliminated from the transmitting end, and the only observable information that can be used is the input power that can be measurable at the transmitting end. 


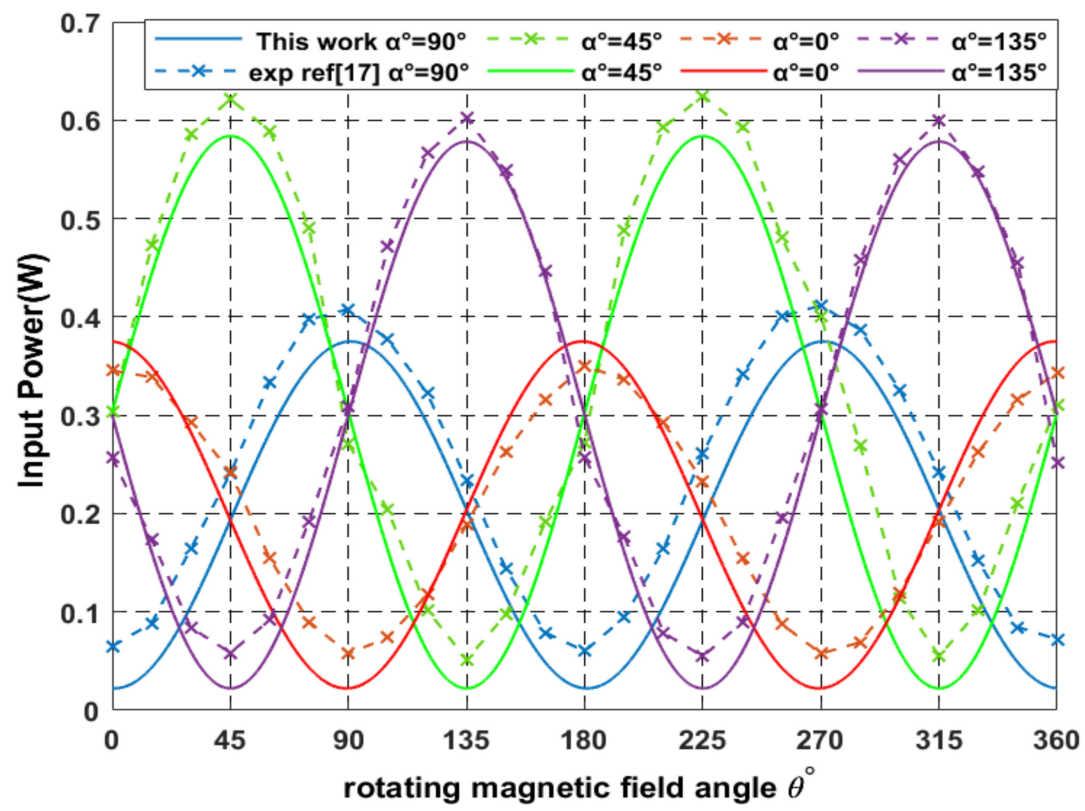

Figure 7. Input power when the receiver is placed at different positions of $\alpha^{\circ}$ in the presence of a rotating magnetic field.

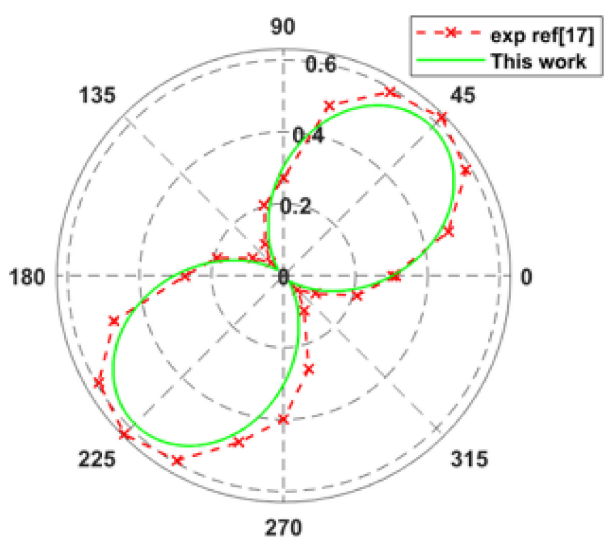

(a)

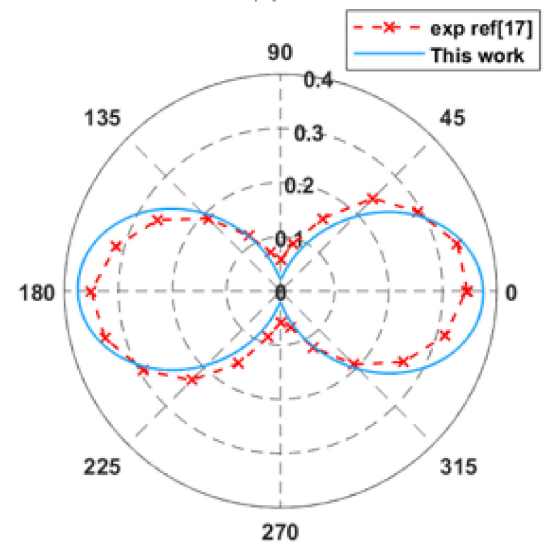

(c)

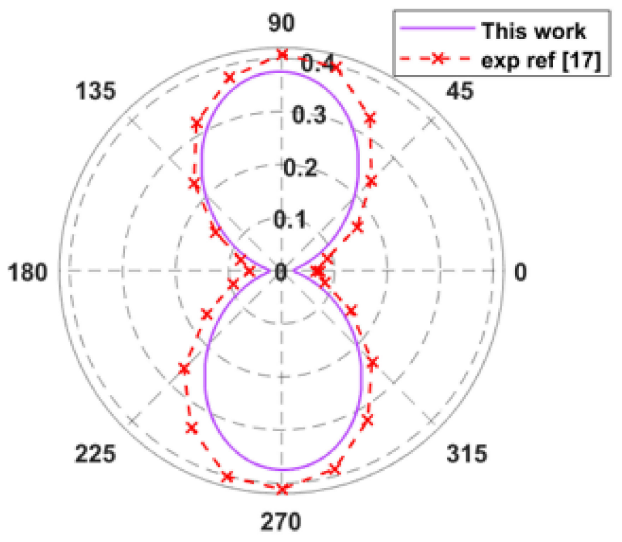

(b)

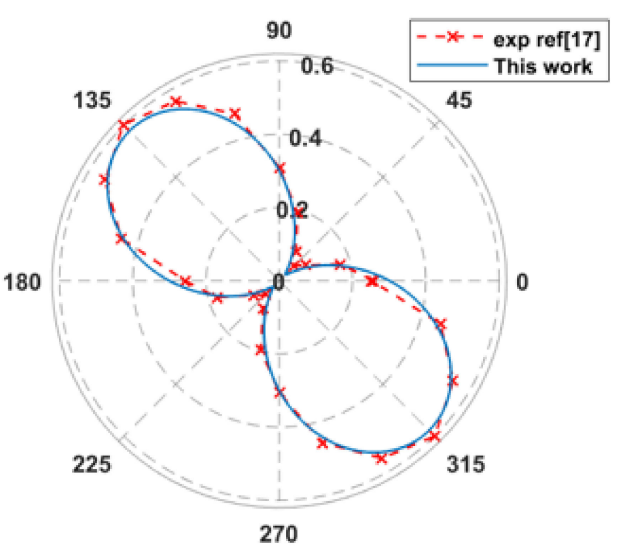

(d)

Figure 8. (a-d) Polar plot of the input power when the receiver is placed at $0^{\circ}, 45^{\circ}, 90^{\circ}$, and $135^{\circ}$, respectively, in the presence of rotating magnetic field when $d=0.3 \mathrm{~m}$. 
The input power and efficiency results established using the proposed model in this paper were benchmarked and compared with the excremental work of [17]. In Figures 7-9, it is noticeable that both results are in close agreement.

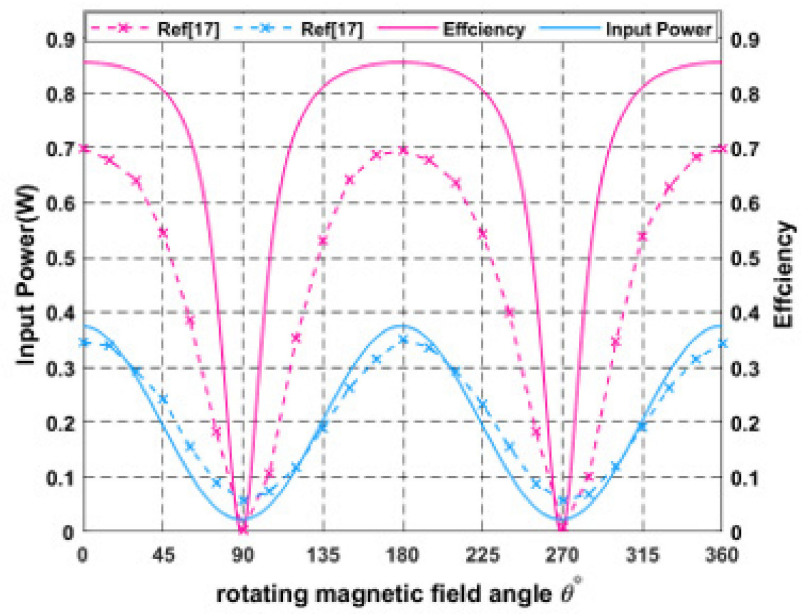

(a)

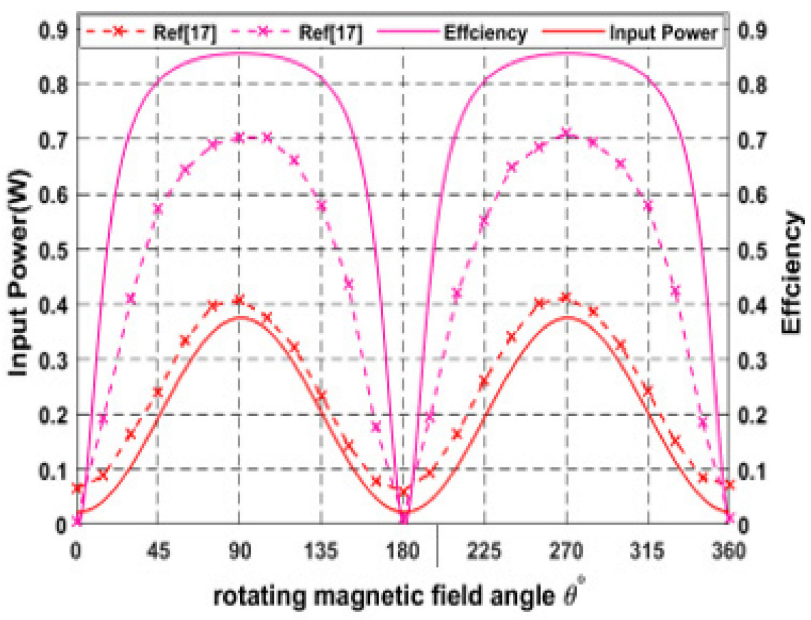

(c)

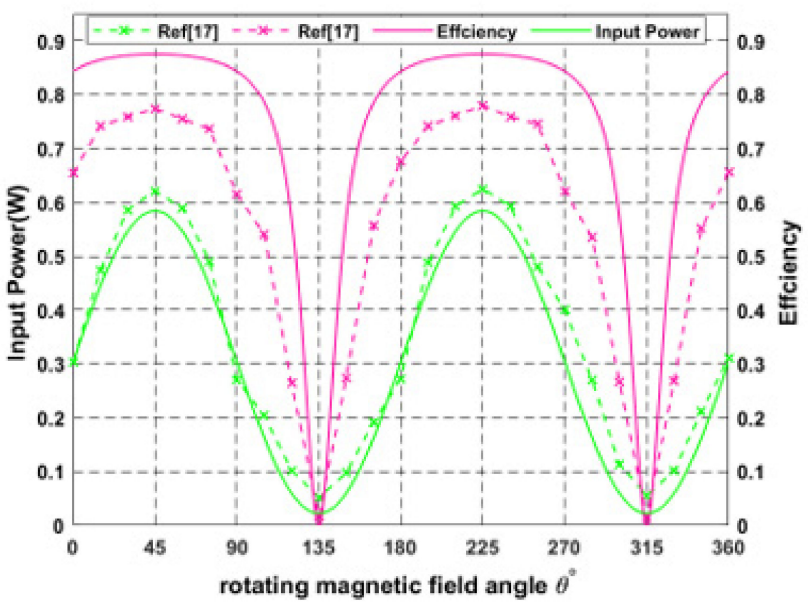

(b)

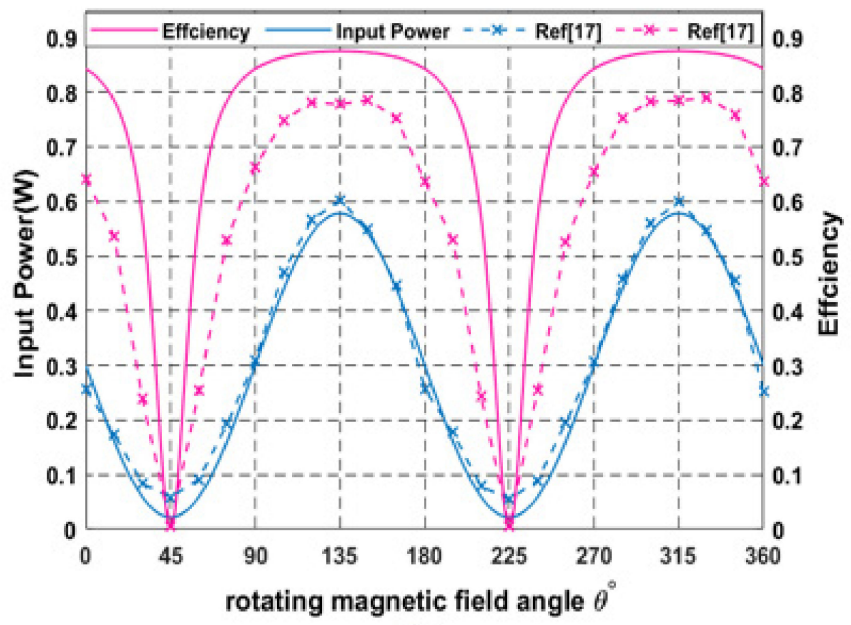

(d)

Figure 9. (a-d) Input power and efficiency plots when the receiver is placed at $0^{\circ}, 45^{\circ}, 90^{\circ}$, and $135^{\circ}$, respectively, in the presence of a rotating magnetic field when $d=0.3 \mathrm{~m}$.

In Figure 10, the input power is shown when $\theta^{\circ}$ and $\alpha^{\circ}$ rotate with the same angle (which means the maximum input power for the 2D WPT system). The used input signal for $\theta^{\circ}$ and $\alpha^{\circ}$ is a ramp with a slope of 180 for $1 \mathrm{~s}$ of simulation time.

Figure 10 represents the summation of the existing maxima input power of the 2D WPT system when the receiver rotates according to an angular function of $\alpha^{\circ}(t)=180 t$ (the velocity of the receiver is the derivative of the function of $\alpha^{\circ}(t)$ ) and where $t$ varies from 0 to $1 \mathrm{~s}$.

By controlling $\theta^{\circ}(t)$ to track the exact function of $\alpha^{\circ}(t)$, we can ensure a continuous charging between two maximum values of $0.38(\mathrm{~W})$ when the receiver is totally coupled with one transmitter $\left(0^{\circ}, 90^{\circ}\right)$ and a value of $0.58(\mathrm{~W})$ when the receiver is coupled equally from both transmitters $\left(45^{\circ}, 135^{\circ}\right)$, The movement of the receiver is continuous with a constant velocity, as is demonstrated in Figure 10. 


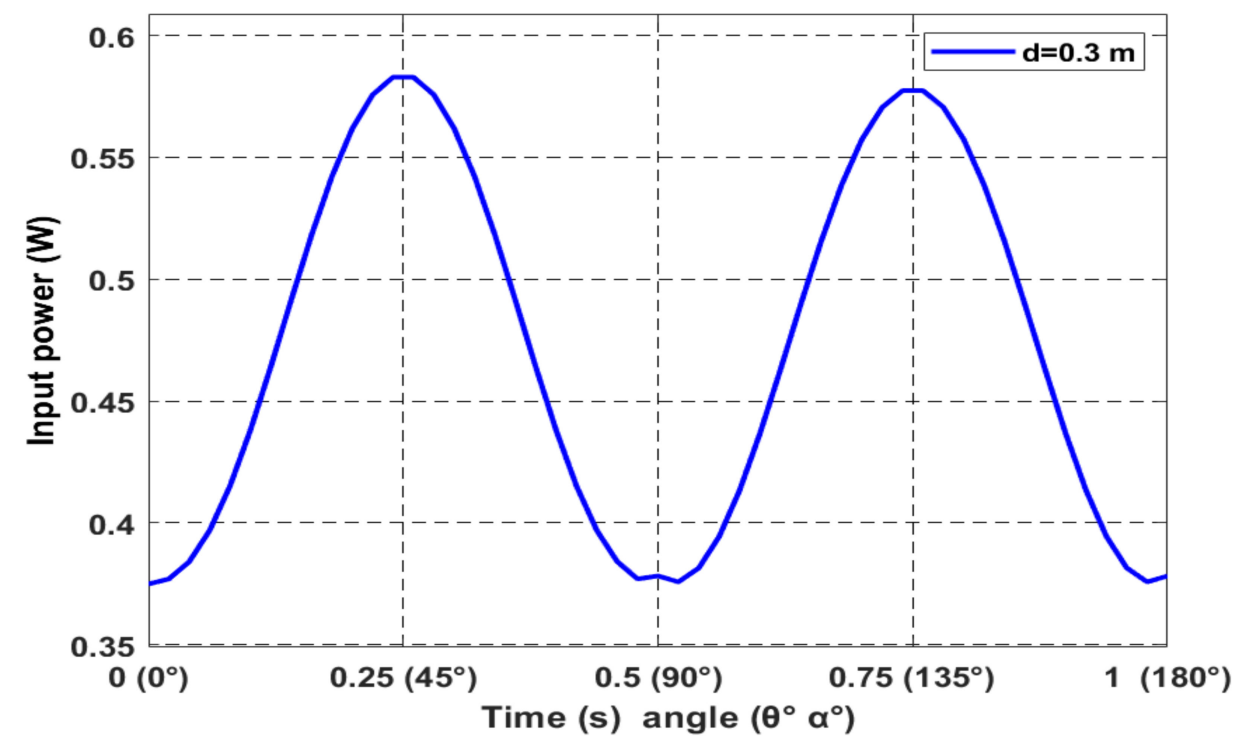

Figure 10. Input power variation versus time when $\theta^{\circ}(t)=\alpha^{\circ}(t)$.

As we can see, the input power reaches its maximum when the receiver and the magnetic resultant are situated in the middle of the transmitting end $\left(45^{\circ}, 135^{\circ}\right)$, in which case the receiver is coupled with two transmitters equally, and the power increases, unlike the $\left(0^{\circ}, 90^{\circ}\right)$ positions, where the power is at its minimum because the receiver is coupled with one transmitter at this time, as evidenced from Figure 5, illustrating the relationship between the mutual coupling and angular variation of the receiver $\alpha^{\circ}$.

Figures 7 and 8 show that the power is omnidirectional and periodic; hence, studying only the first quarters is enough for simulating the control process of the two-dimensional WPT system.

\section{Methodology and Implementation}

\subsection{The Extremum Seeking Control Implementation for 2D WPT System}

According to (14), it is possible to maximize the load power through the input power. To maximize the power transfer efficiency, the only available feedback from the transmitter is the input power. Thus, taking it as the objective function $f(\theta)$ for the extremum seeking controller (ESC) as follows:

$$
f(\theta)=R I^{2}+\left(R_{3}+R_{L}\right) \frac{w^{2} I^{2}}{\left(R_{3}+R_{L}\right)^{2}+X_{3}^{2}} \times\left(M_{13}(\alpha) \cos \theta+M_{23}(\alpha) \sin \theta\right)^{2}
$$

The continuously differentiable function $f(\theta)$ can be approximated locally by Equation (15), as detailed in [20]:

$$
f(\theta)=f^{*}+\frac{f^{\prime \prime}}{2}\left(\theta-\theta^{*}(t)\right)^{2}
$$

where $f$ is a $C^{2}$ function, $\theta$ is the input, and $\theta^{*}$ is the optimal value that renders the output equal to the extremum $f^{*}$.

The objective is to minimize the difference between the unknown optimal input value and the current value, so that $\theta$ converges to $\theta^{*}$ which makes $f(\theta)=f^{*}$. The fact that the optimum $\theta^{*}(t)$ is a variable function of time due to the receiver dynamic mobility function $\alpha(t)$. Therefore, in this case, we use the single-parameter extremum seeking a dynamic plant.

Since the receiver is under continuous movement, our plant function will have one control input variable, $\theta(t)$, to control the magnetic field resultant orientation and two other plant variables, $M_{13}(\alpha)$ and $M_{23}(\alpha)$, to simulate the receiver movement. By the virtue 
of Equations (7) and (8), we can directly inject any type of rotational movement function for the receiver angle $\alpha$, including the trajectory and velocity, and observe how the controller tracks the maximum power, as illustrated in Figure 11.

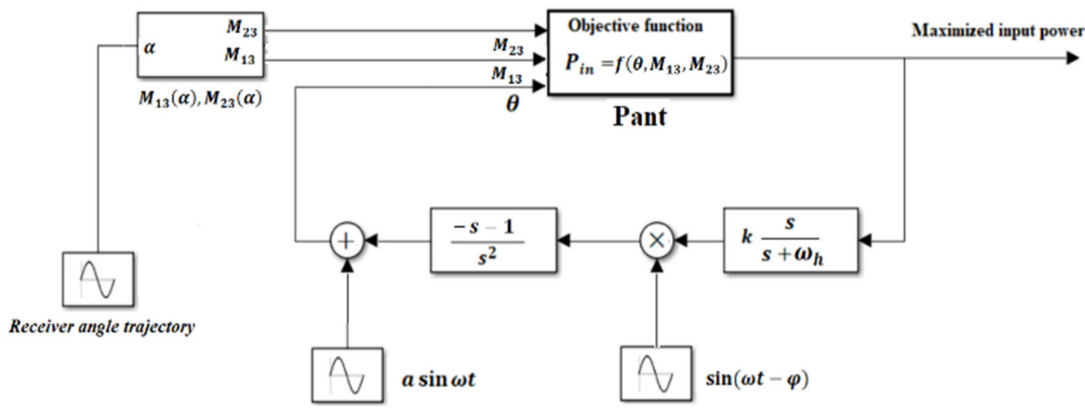

Figure 11. Extremum seeking scheme for 2D omnidirectional WPT power maximization.

The controller starts to find the optimum value of the input $\theta$ that maximizes the objective function regardless of the mobility behavior of the receiver. Since the power transfer is omnidirectional, the magnetic field angle $\theta$ control process is run only in the first two quarters. Consequently, it will cover the other remaining two.

The control feedback consists of a second-order integrator $\frac{1}{s^{2}}$, which is present because the optimum value of the input power is varying with respect to time due to the receiver mobility, while the term $-(s+1)$ is added to reduce the relative degree of the loop. The high-pass filter $\frac{s}{s+\omega_{h}}$ cutoff frequency $\omega_{h}$ is tuned so that the system in a closed loop satisfies the stability condition $k$, a proportional gain that increases the speed response of the system, while $a \sin (\omega t)$ is a perturbation signal with a frequency $\omega$ higher than the receiver velocity, and the perturbation amplitude $a$ must be sufficiently large enough to ensure a steady-state output error. The phase shift parameter $\phi$ is used for fine tuning after setting all the ESC parameters.

The simulation results are obtained by running Simulink based on the designed ES scheme, as shown in Figure 11, which shows an accurate tracking of the maximum with a small time response for both steady-state and continuous rapid movement of the receiver.

In Figure 12 the controller can track the angle of the receiver with a response time of $10 \mu \mathrm{s}$, and it reaches the maximum of the power transfer in almost $10 \mu$ s of response time. In this case, the receiver is installed in a steady-state position of $45^{\circ}$.
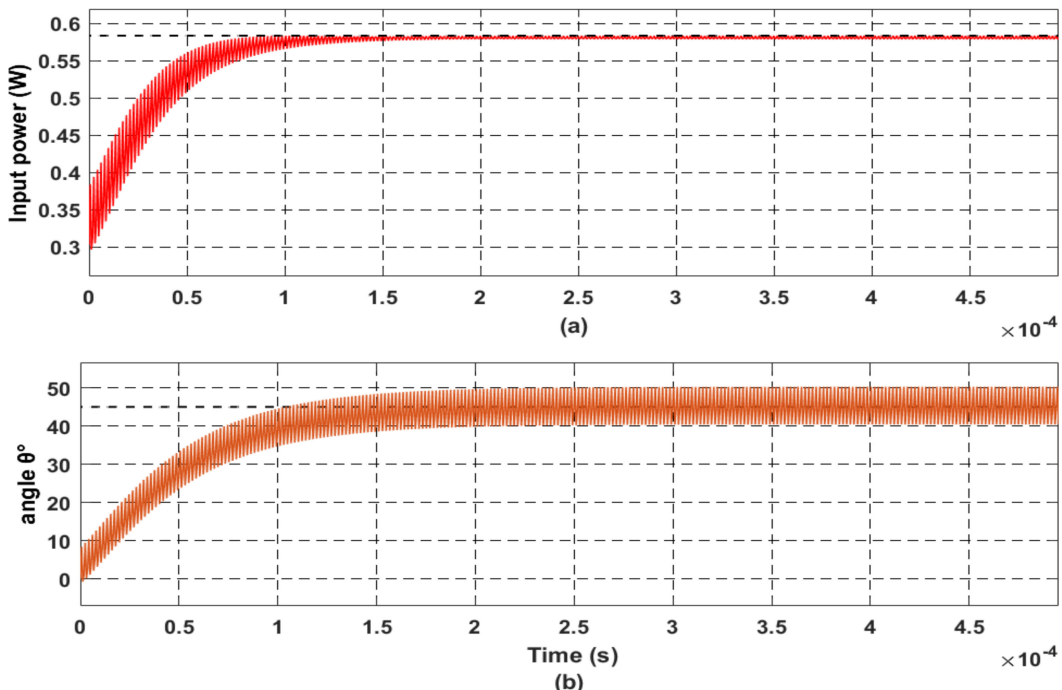

Figure 12. (a) Input power in the closed loop when the receiver is in a static position of $45^{\circ}$ and the distance $d=0.3 \mathrm{~m}$. (b) Controlled resultant magnetic field angle $\theta$. 
This input angle signal in Figure 13a simulates a movement at a fixed angular speed of $23.562 \mathrm{rad} / \mathrm{s}$ from $0 \mathrm{~s}$ to $0.1 \mathrm{~s}$. Having obtained a steady state, the receiver acquires from $0.1 \mathrm{~s}$ to $0.2 \mathrm{~s}$. The movement sequence is repeated, as demonstrated in the angular receiver position diagram in Figure 13a.

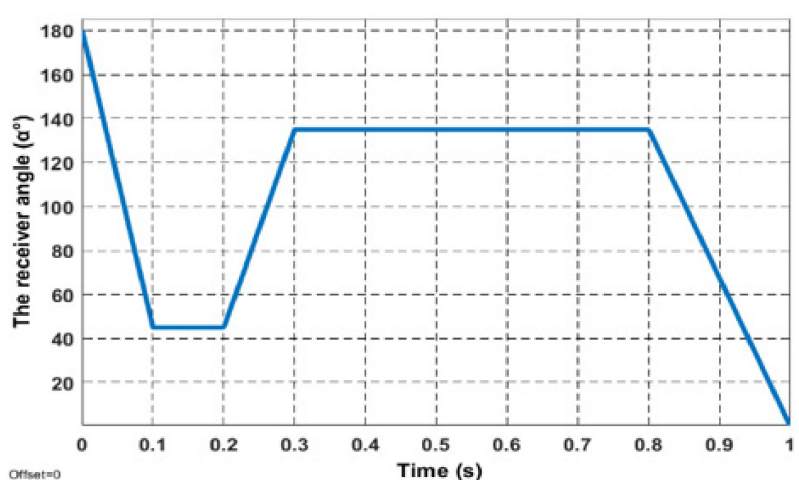

(a)

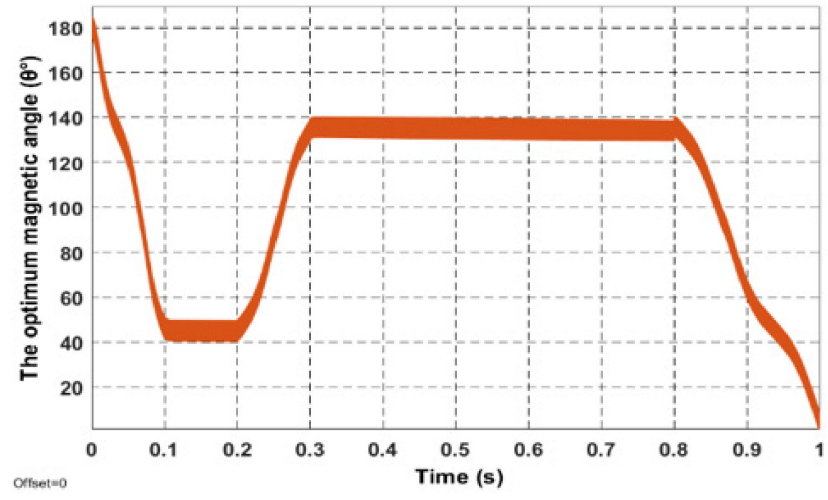

(b)

Figure 13. (a) Used signal for simulating a specific rotational motion of the receiver. (b) Optimum angle $\theta$ searched by the controller.

The signal in Figure 13b represents the optimum control command for power extremization, which was searched by the ES controller, demonstrating how the resultant magnetic field tracks ideally perfectly the angular position of the receiver.

\subsection{The Impact of Receiver Distance Variation on ES Controller}

The rotational motion of the receiver is simulated for different values of distance $d$, showing that the controller is able to track the maximum of power over different distances, using the same filters parameter.

Using the same steps as those of the previous section, the values of mutual coupling versus the distance variations are shown in Figure 14.

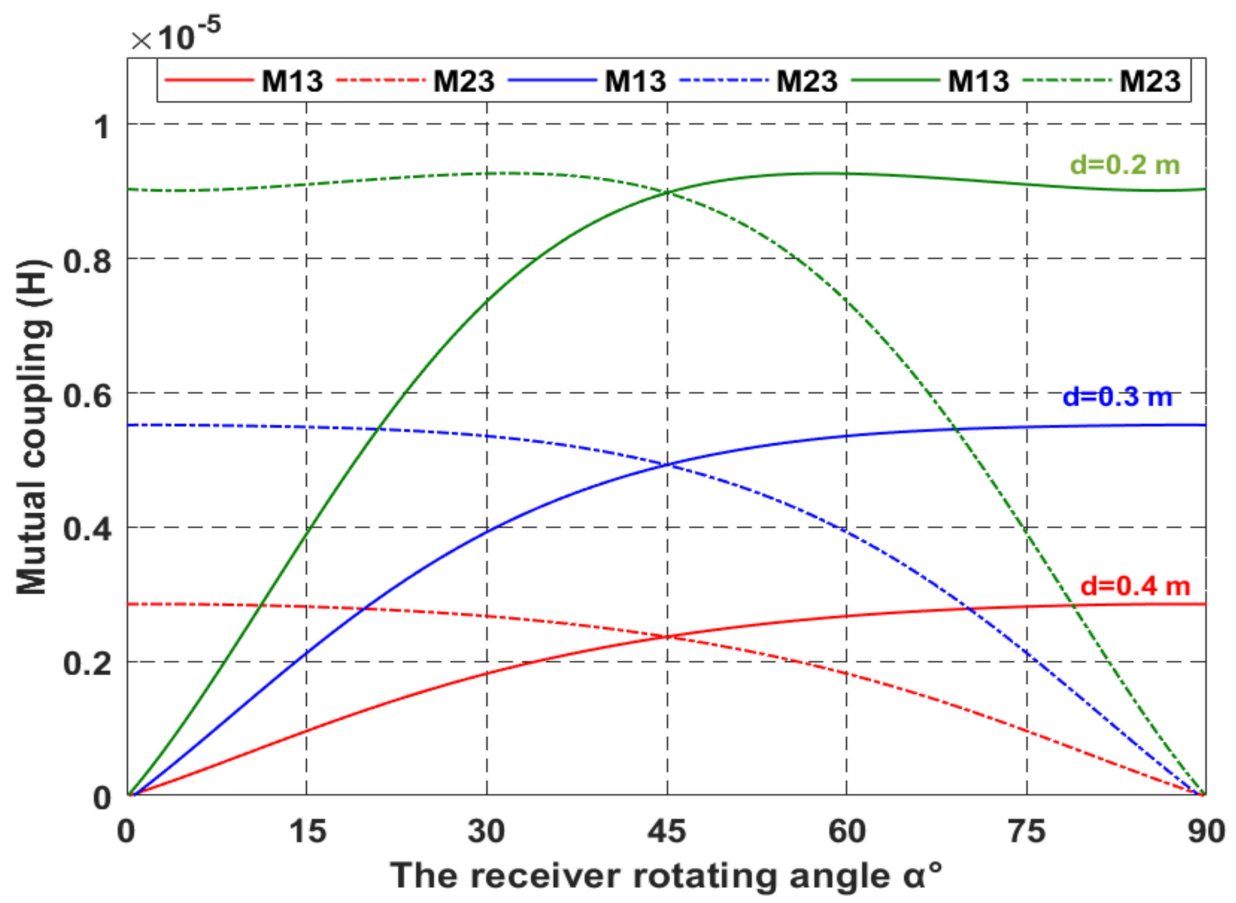

Figure 14. Mutual coupling curves in terms of $\alpha$ for different values of distance. 
From the above figure, it is noticeable that an increase in the distance $d$ between the receiver and the transmitter will attenuate the mutual coupling, as well as the wireless power transfer efficiency. Further, we notice that the plot of any given distance meets that of the corresponding mutual coupling plot at $45^{\circ}$, the point of maximum power tracking. By taking the input angle function $\alpha(t)=18,000 t$, we can simulate a load rotation from $0^{\circ}$ to $180^{\circ}$ with an angular velocity of $314 \mathrm{rad} / \mathrm{s}$.

Figure 15 clearly shows the fact that the maximum input power function of the two-dimensional WPT system when $\theta(t)=\alpha(t)=18,000 t$ decreases when the distance $d$ increases.
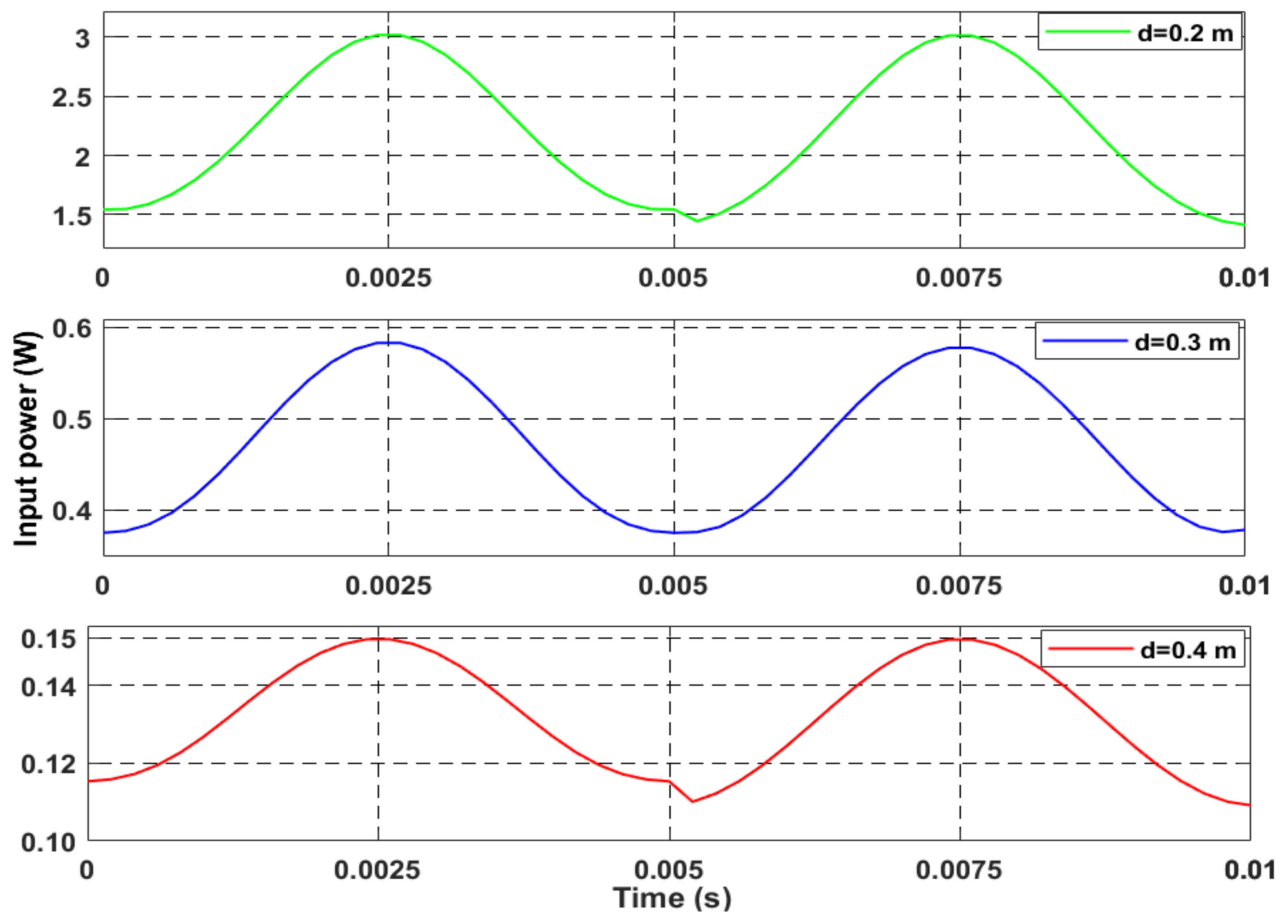

Figure 15. Maximum Input power variation with respect to time when $\theta^{\circ}(t)=\alpha^{\circ}(t)$ and the distance $d$ is varied from $0.2 \mathrm{~m}$ to $0.4 \mathrm{~m}$.

\section{Results and Benchmarking}

\subsection{Results and Benchmarking for the ESC Method in 2D WPT System}

Table 1 shows the simulation parameters and ESC system settings. In Figure 16a, the curve in the dashed black line represents the calculated maximum power when the receiver rotates according to the trajectory suggested in Figure 13a. The red curve represents the maximized input power when the closed loop ES control action is applied.

Table 1. Extremum seeking control parameters and system settings.

\begin{tabular}{cc}
\hline Extremum Seeking Control Parameters & Expression \\
\hline Reductive relative degree factor & $-(s+1)$ \\
\hline High-pass filter & $\frac{s}{s+\omega_{h}}$ \\
\hline Perturbation signal & $a \sin (\omega t)$ \\
\hline Proportional gain & $k$ \\
\hline
\end{tabular}




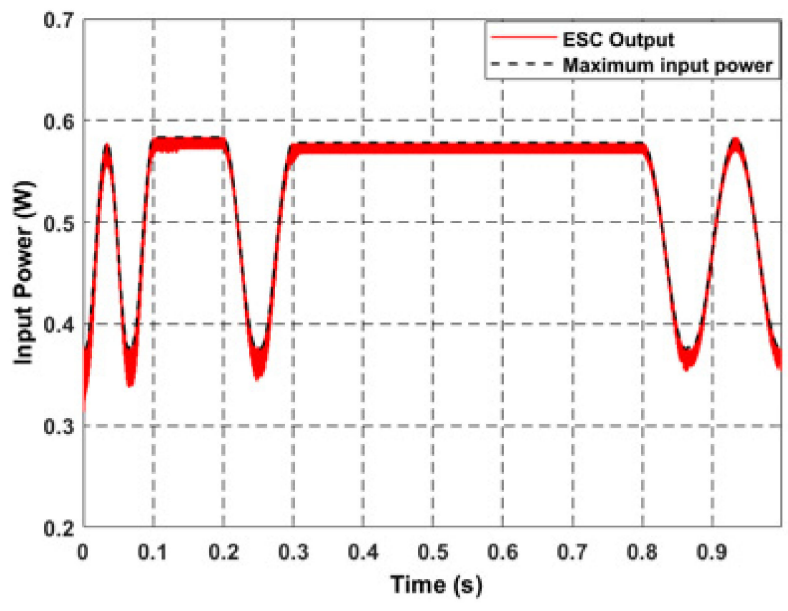

(a)

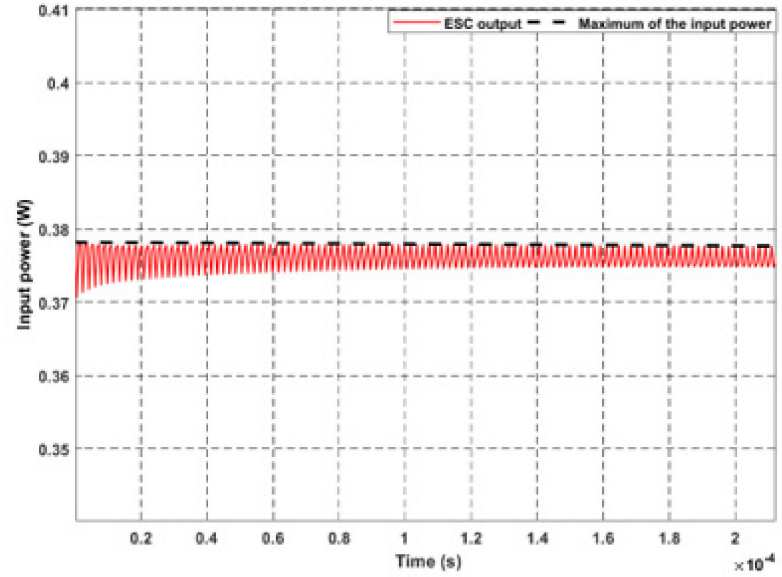

(b)

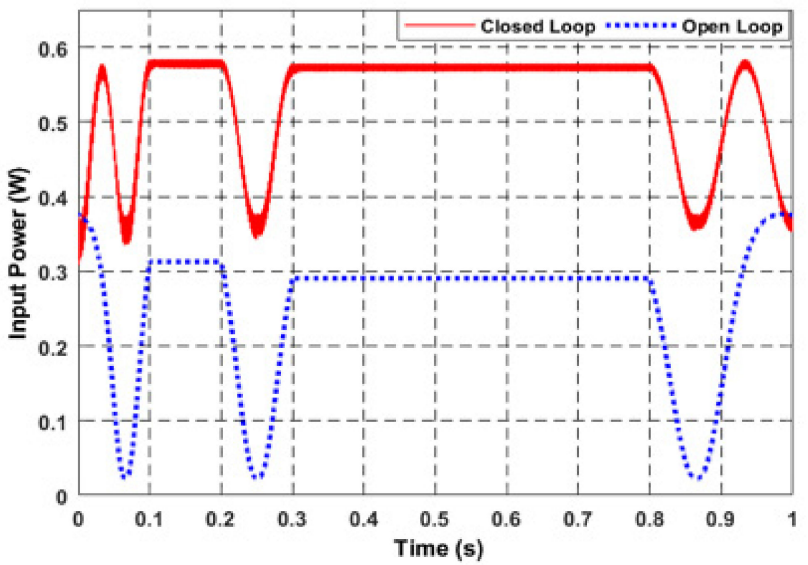

(c)

Figure 16. (a) Maximized input power response in the closed loop versus the calculated input power. (b) Zoomed view showing how the input power in the closed loop converges to the maximum. (c) Input power comparison between the closed loop and the open loop.

From Figure 16, one can observe that the receiver will pick up the maximum power regardless of its position or speed. Hence, the maximization of the power transfer for the 2D orthogonal coils system is achieved in both cases of rapid mobility and static load. Figure $16 \mathrm{~b}$ also shows that the 2D-WPT system is able to achieve its maximum power output in less than $10 \mu$ s under the ESC closed loop, and it outperforms the other used algorithms reported in [17] with an almost insignificant static error.

\subsection{Results and Benchmarking for the ESC Method under Distance Variation}

Figure 17 shows how the controller can accurately track each maximum input power function at different distances without recalibrating its filter parameters. The controller can propel the system to reach its maximum power transfer even when the angular velocity of the receiver is in the range of $314 \mathrm{rad} / \mathrm{s}$, and thus, there is a possibility of using the ESC to power rotating loads such as drones. Depending on the function $\alpha(t)$ injected into the system the controller will adjust the magnetic resultant angle $\theta(t)$ to the optimum value, which will render the output fixed to the maximum regardless of the variation of the receiving angle and its distance in time. 


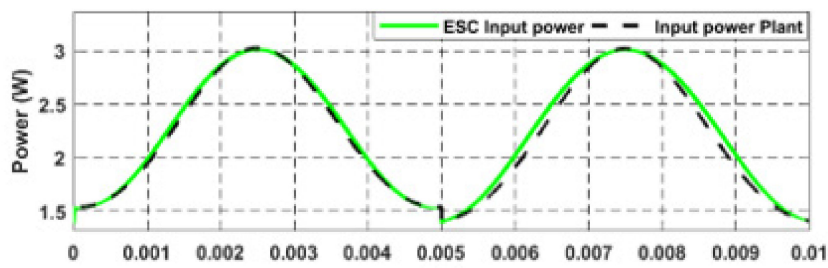

(a)

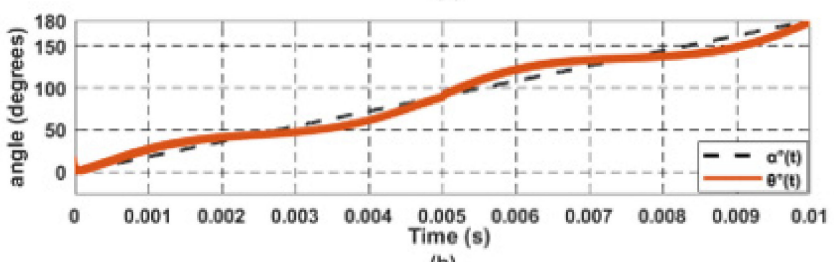

(b)
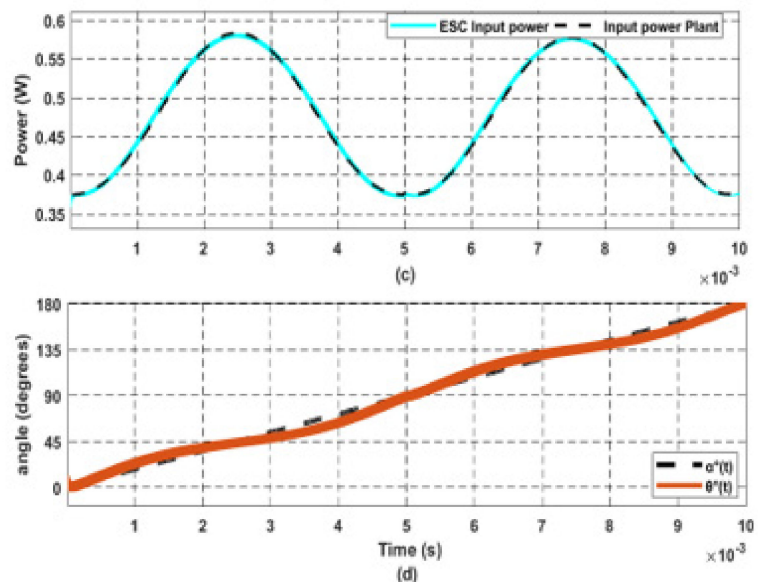

$d=0.3 \mathrm{~m}$
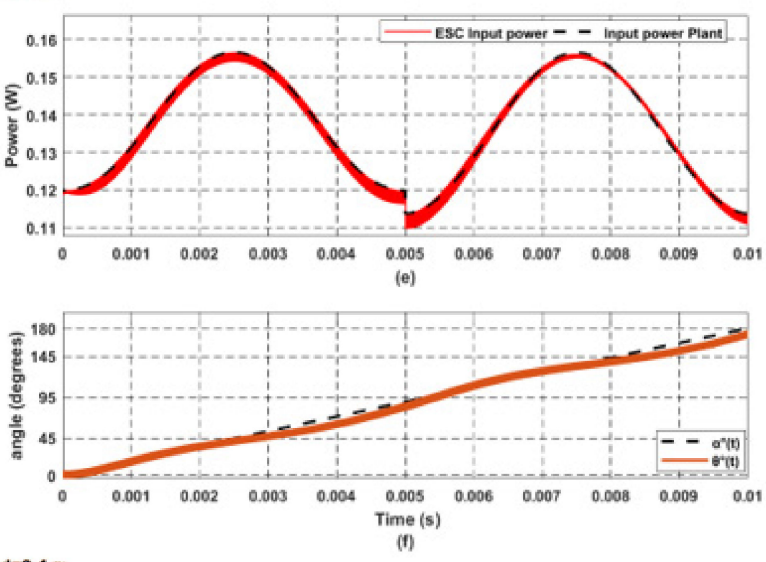

$d=0.4 \mathrm{~m}$

Figure 17. (a,c,e) ESC maximization of the input power for different values of distance of $(\mathbf{b}, \mathbf{d}, \mathbf{f})$. ESC of the magnetic resultant angle $\theta$ optimization for different values of $d$.

In Figures 18 and 19, we simulate a continuous distance variation of the load from $0.2 \mathrm{~m}$, which is the minimum realizable distance for the model until $5 \mathrm{~m}$, where the receiver angle is fixed at $45^{\circ}$ and $0^{\circ}$.

It is noticeable that the system in the closed loop can track the maximum of input power even when the receiver moves away from the transmitting end continuously and its angular position is fixed at $45^{\circ}$ and $0^{\circ}$. Figures $18 \mathrm{c}$ and $19 \mathrm{c}$ show that the optimum magnetic field resultant angle $\theta$ is controlled to be constant at the exact angular position of the receiver even when the distance is varying continuously.

From Section 4, we can conclude that the ESC controller can reach evaluable maximum power whether the receiver rotates in continuous movement or in a continuous distance variation. The simulation of the mutual coupling in terms of $\alpha(t)$ via Ansys serves to design the controller and observe its response for research purposes. Once the controller is installed, it will not need any information on the receiver; it will track only the input power signal and maximize it.

By changing the design parameters of the coils, it is possible to increase the power delivered to the load, and the parameters used to simulate the system in this research are those in the benchmarking paper [17]. However, in this paper, more information is provided about the mutual coupling relationship and the angle of rotation of the receiver $\alpha$. It also takes into consideration the problem of mobility and receiver velocity, as well as the distance variation in contrast to [17], which only focuses on localizing the fixed position of the receiver after calibration operations. The input power calculation based on the Ansys software simulation in this work matches the experimental results in [17]. This paper utilizes an efficient control technique that relies on adaptive control theory 
to find the maximum output of an objective function without sensing the reference set point. The work continues extending the same ideas for battery charging purposes and the powering of moving loads benefiting from concepts presented in recently reported work $[21,22]$. The currently reported work shows omnidirectional approaches under high transmitter-receiver misalignment conditions [23].

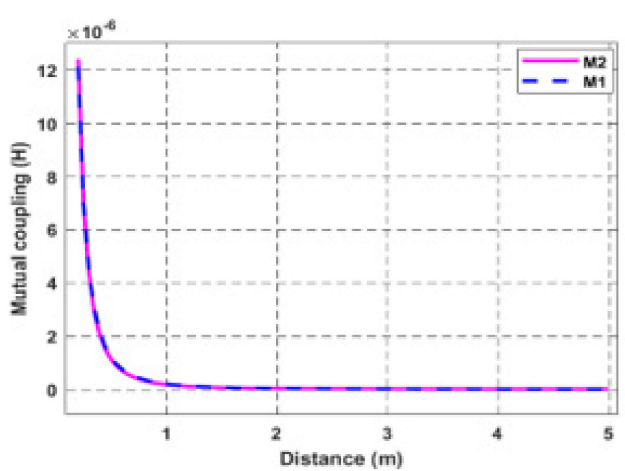

(a)

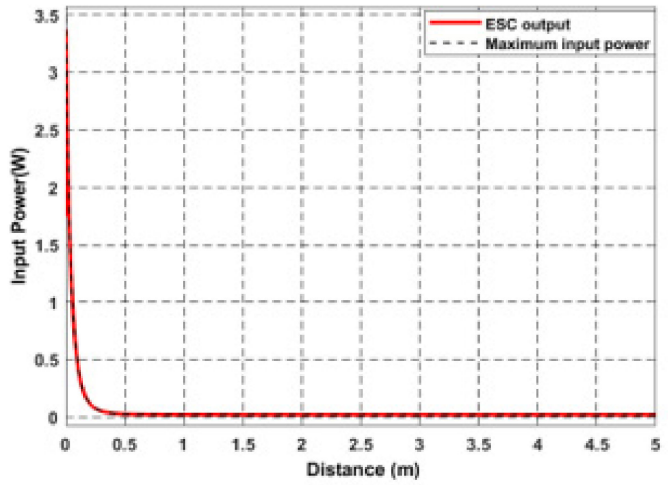

(b)

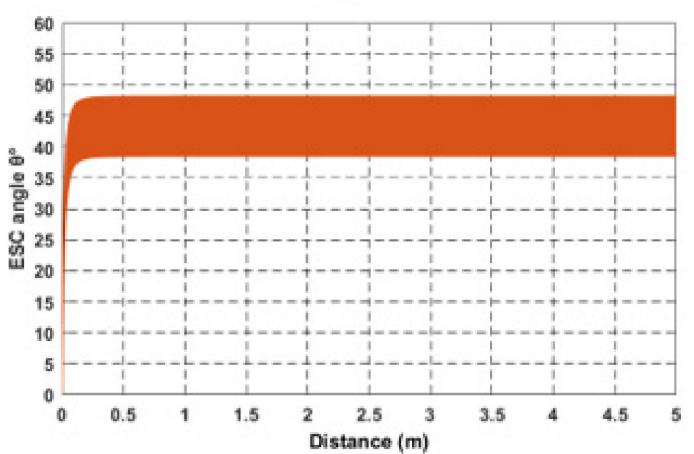

(c)

Figure 18. Continuous distance variation of the receiver when $\alpha$ is fixed at $45^{\circ}$. (a) Mutual coupling in terms of the receiver distance variation. (b) Input power maximum tracking under a continuous distance variation. (c) Optimum magnetic resultant angle $\theta$ searched by the ESC when the distance is varying continuously.

\subsection{Discussion of Proposed WPT System Operational Scenarios and Its Limitations}

The drone battery energy limitation is a major constraint in drone path planning during routine operation, as it curbs long surveillance missions. To resolve this issue, recharging stations can be introduced where the drone can autonomously land to recharge its battery before continuing the mission [24]. In our proposed model, we foresee a drone landing on a supervised surface or hovering around a circular disk surrounding the charging station. The drone must maintain a distance not more than $0.3 \mathrm{~m}$ away from the transmitting end. This will enable the drone to maximize its received energy efficiency and reduce its charging time. The charging distance can be increased further by using larger transmitting coils.

The model does not assume a special power loading mode. However, the drone velocity plays an important role in determining the charging efficiency and time. If the drone is made to hover in a circular movement on a disk surrounding the charging station, the ESC controller will track its movement. The ESC tracking system facilitates convergence to a maximum power transfer within a brief period of time, provided that the drone is in close proximity in the order of $0.3 \mathrm{~m}$ from the transmitter, and the ESC controller tracking speed has to be always higher than the drone speed.

Some real-life drone applications may require drones to move freely in a 3D sphere instead of a 2D disk trajectory and some emergency scenarios or routine operations in 
hazardous environments dictates that the drone can only hover during the charging process. This makes our 2D model only partially realistic due to restrictions of the aerial drone movement trajectory. However, we are currently devolving a fully realistic 3D model that takes care of the jerky movement of aerial drones during the hovering process, stormy weather conditions, etc. and its details will be the subject of a future paper.

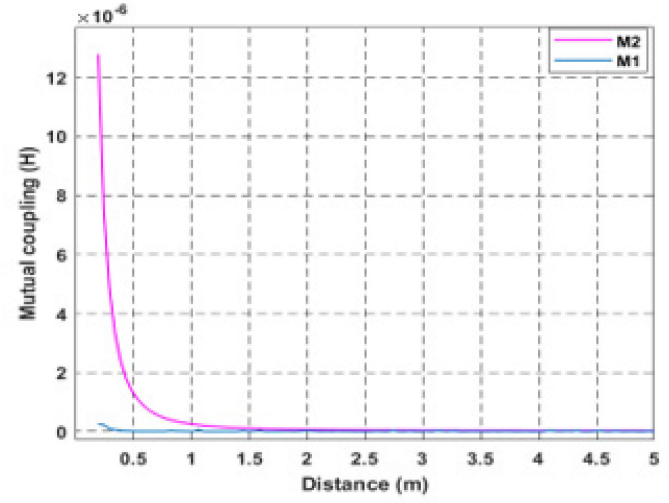

(a)

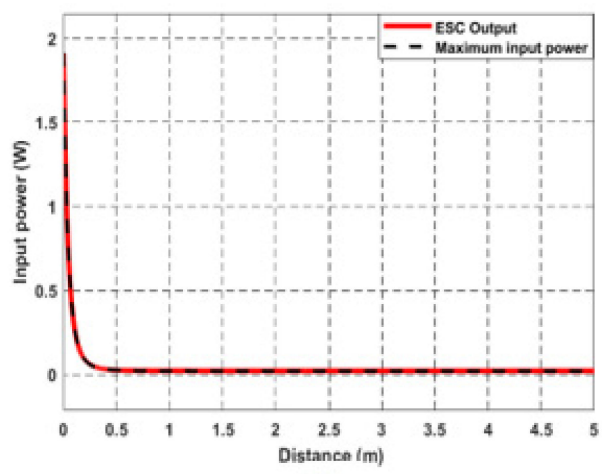

(b)

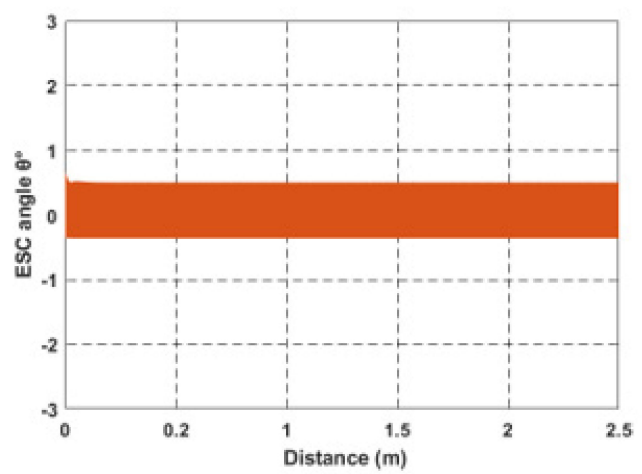

(c)

Figure 19. Continuous distance variation of the receiver when $\alpha$ is fixed at $0^{\circ}$. (a) Mutual coupling in terms of the receiver distance variation $d$. (b) Input power maximum tracking under a continuous distance variation. (c) Optimum magnetic resultant angle $\theta$ searched by the ESC when the distance is varying continuously.

\section{Conclusions}

This paper presented a two-dimensional wireless power transfer system for a mobile load. The system was explained by illustrating it through its equivalent electric circuit modeling, substituting the orthogonal coils with mutual coupling terms. For realization purposes, a comprehensive model was developed that included all the important parameters of the system while dispensing the conditions of mutual coupling to simulate outputs based on the desired inputs. The experimental results in [17] are in close agreement with the theoretical derivations and Ansys simulation results. By analyzing the behavior of input power versus load mobility, an extremum control system that seeks to maximize wireless power transmission under high-speed receiver motion was proposed. Furthermore, the control unit was tested at various distances, including the load movement and speed. Finally, the performance of the controller was validated in the proposed benchmarking paper, as both results matched precisely. The input power converges with the calculated maximum of $0.6 \mathrm{~W}$ in $10 \mu \mathrm{s}$ when the receiver is in a $45^{\circ}$ static position for $d=0.3 \mathrm{~m}$. When the receiver rotates, the maximum power is guaranteed for an angular velocity of $314.159 \mathrm{rad} / \mathrm{s}$. In theory, the control method is suitable for smart applications such as drones and smart sensors, as the Extremum seeking control is effective in terms of response time in addition to steady-state error providing a maximum power transfer regardless of the position of the receiver, trajectory, or speed. 
Author Contributions: Conceptualization, methodology, and writing, O.A., M.H.H. and S.K.; software, O.A.; validation and revision: M.H.H., S.K., E.A.A.E. and F.E.M.S., funding: M.H.H. and E.A.A.E. and F.E.M.S. All authors have read and agreed to the published version of the manuscript.

Funding: The authors extend their appreciation to the Deanship of Scientific Research at King Khalid University for funding this work through the Research Group Program under Grant Number (R.G.P.1/219/42). This work has also been partially sponsored by International Islamic University Malaysia, Publication Research Initiative Grant Scheme Number P-RIGS18-003-0003.

Conflicts of Interest: The authors declare no conflict of interest.

\section{References}

1. Cetinkaya, O.; Balsamo, D.; Merrett, G.V. Internet of MIMO Things: UAV-Assisted Wireless-Powered Networks for Future Smart Cities. IEEE Internet Things Mag. 2020, 3, 8-13. [CrossRef]

2. Rabello, A.; Brito, R.C.; Favarim, F.; Weitzenfeld, A.; Todt, E. Mobile System for Optimized Planning to Drone Flight applied to the Precision Agriculture. In Proceedings of the 2020 3rd International Conference on Information and Computer Technologies (ICICT), San Jose, CA, USA, 9-12 March 2020; pp. 12-16.

3. Xiao, W.; Li, M.; Alzahrani, B.; Alotaibi, R.; Barnawi, A.; Ai, Q. A Blockchain-Based Secure Crowd Monitoring System Using UAV Swarm. IEEE Netw. 2021, 35, 108-115. [CrossRef]

4. Vahidi, V.; Saberinia, E.; Morris, B.T. OFDM Performance Assessment for Traffic Surveillance in Drone Small Cells. IEEE Trans. Intell. Transp. Syst. 2019, 20, 2869-2878. [CrossRef]

5. Busnel, Y.; Caillouet, C.; Coudert, D. Self-Organized Disaster Management System by Distributed Deployment of Connected UAV. In Proceedings of the 2019 International Conference on Information and Communication Technologies for Disaster Management (ICT-DM), Paris, France, 18-20 December 2019.

6. Wu, J.; Dai, X.; Gao, R.; Jiang, J. A Coupling Mechanism With Multidegree Freedom for Bidirectional Multistage WPT System. IEEE Trans. Power Electron. 2021, 36, 1376-1387. [CrossRef]

7. Tian, X.; Chau, K.T.; Liu, W.; Lee, C.H.T. Analysis of Multi-Coil Omnidirectional Energy Harvester. IEEE Trans. Magn. 2021, 57, 1-6. [CrossRef]

8. $\quad$ Rong, C.; He, X.; Liu, M.; Wang, Y.; Liu, X.; Lu, C.; Zeng, Y.; Liu, R. Omnidirectional Free-Degree Wireless Power Transfer System Based on Magnetic Dipole Coils for Multiple Receivers. IEEE Access 2021, 9, 81588-81600. [CrossRef]

9. Han, H.; Mao, Z.; Zhu, Q.; Su, M.; Hu, A.P. A 3D Wireless Charging Cylinder With Stable Rotating Magnetic Field for Multi-Load Application. IEEE Access 2019, 7, 35981-35997. [CrossRef]

10. Feng, J.; Li, Q.; Lee, F.C.; Fu, M. Transmitter Coils Design for Free-Positioning Omnidirectional Wireless Power Transfer System. IEEE Trans. Ind. Inform. 2019, 15, 4656-4664. [CrossRef]

11. Zhang, Z.; Zhang, B.; Tong, R.; Ai, W.; Chang, S.; Wang, J. Optimal Design of Quadrature-Shaped Pickup for Omnidirectional Wireless Power Transfer. IEEE Trans. Magn. 2018, 54, 1. [CrossRef]

12. Kisseleff, S.; Akyildiz, I.F.; Gerstacker, W. Beamforming for magnetic induction based wireless power transfer systems with multiple receivers. In Proceedings of the 2015 IEEE Global Communications Conference (GLOBECOM), San Diego, CA, USA, 6-10 December 2015; pp. 1-7.

13. Wang, H.; Zhang, C.; Yang, Y.; Liang, H.W.R.; Hui, S.Y.R. A Comparative Study on Overall Efficiency of Two-Dimensional Wireless Power Transfer Systems Using Rotational and Directional Methods. IEEE Trans. Ind. Electron. 2022, 69, 260-269. [CrossRef]

14. Lim, Y.; Park, J. A Novel Phase Control based Energy Beamforming Techniques in Non-radiative Wireless Power Transfer. IEEE Trans. Power Electron. 2014, 30, 1. [CrossRef]

15. Lin, D.; Zhang, C.; Hui, S.Y.R. Mathematic Analysis of Omnidirectional Wireless Power Transfer-Part-II Three-Dimensional Systems. IEEE Trans. Power Electron. 2016, 32, 613-624. [CrossRef]

16. Zhang, C.; Lin, D.; Hui, S.Y. Basic Control Principles of Omni-Directional Wireless Power Transfer. IEEE Trans. Power Electron. 2015, 31, 1. [CrossRef]

17. Lin, D.; Zhang, C.; Hui, S.Y.R. Mathematical Analysis of Omnidirectional Wireless Power Transfer-Part-I: Two-Dimensional Systems. IEEE Trans. Power Electron. 2016, 32, 625-633. [CrossRef]

18. Su, M.; Liu, Z.; Zhu, Q.; Hu, A.P. Study of Maximum Power Delivery to Movable Device in Omnidirectional Wireless Power Transfer System. IEEE Access 2018, 6, 76153-76164. [CrossRef]

19. Lin, D.; Hui, S.Y.R.; Zhang, C. Omni-directional wireless power transfer systems using discrete magnetic field vector control. In Proceedings of the 2015 IEEE Energy Conversion Congress and Exposition (ECCE), Montreal, QC, Canada, 20-24 September 2015; pp. 3203-3208.

20. Ariyur, K.B.; Krstić, M. Real-Time Optimization by Extremum-Seeking Control; Wiley: New York, NY, USA, 2003.

21. Xu, C.; Zhuang, Y.; Song, C.; Huang, Y.; Zhou, J. Dynamic Wireless Power Transfer System With an Extensible Charging Area Suitable for Moving Objects. IEEE Trans. Microw. Theory Tech. 2021, 69, 1896-1905. [CrossRef]

22. Babaki, A.; Vaez-Zadeh, S.; Zakerian, A.; Covic, G.A. Variable-Frequency Retuned WPT System for Power Transfer and Efficiency Improvement in Dynamic EV Charging With Fixed Voltage Characteristic. IEEE Trans. Energy Convers. 2021, 36, $2141-2151$. [CrossRef] 
23. Dong, Z.; Li, X.; Liu, S.; Xu, Z.; Yang, L. A Novel All-Direction Antimisalignment Wireless Power Transfer System Designed by Truncated Region Eigenfunction Expansion Method. IEEE Trans. Power Electron. 2021, 36, 12456-12467. [CrossRef]

24. Fendji, J.L.E.K.; Bayaola, I.K.; Thron, C.; Fendji, M.D.; Förster, A. Cost-Effective Placement of Recharging Stations in Drone Path Planning for Surveillance Missions on Large Farms. Symmetry 2020, 12, 1661. [CrossRef] 